\title{
Continuous-spontaneous-localization scalar-field relativistic collapse model
}

\author{
Daniel Bedingham $\odot^{1, *}$ and Philip Pearle ${ }^{2, \dagger}$ \\ ${ }^{1}$ Department of Physics, Royal Holloway, University of London, Egham, YW20 OEX, United Kingdom \\ ${ }^{2}$ Emeritus, Department of Physics, Hamilton College, Clinton, New York 13323, USA
}

(Received 27 June 2019; published 22 October 2019)

\begin{abstract}
The continuous-spontaneous-localization dynamical collapse structure, adapted to the relativistically invariant model where the collapse-generating operator is a one-dimensional scalar field $\hat{\phi}(x, t)$ (mass $m$ ) is discussed. A complete solution for the density matrix is given, for an initial state $|\psi, 0\rangle=\frac{1}{\sqrt{2}}[|L\rangle+|R\rangle]$ when the Hamiltonian $\hat{H}$ is set equal to 0 , and when $\hat{H}$ is the free field Hamiltonian. Here $|L\rangle,|R\rangle$ are coherent states which represent clumps of particles, with mean particle number density $N \chi_{i}^{2}(x)$, where $\chi_{1}(x), \chi_{2}(x)$ are Gaussians of width $\sigma \gg m^{-1}$ with mean positions separated by distance $\gg \sigma$. It is shown that, with high probability, the solution for $\hat{H}=0$ (identical to the short time solution for $\hat{H} \neq 0$ ) favors collapse toward eigenstates of the scalar field whose eigenvalues are close to $\sim \chi_{i}(x)$. Thus, this collapse dynamics results in essentially one clump of particles. However, eventually particle production dominates the density matrix since, as is well known, the collapse generates energy/sec-volume of every particle momentum in equal amounts. Because of the particle production, this is not an experimentally viable physical theory but, as is emphasized by the discussion, it is a sound relativistic collapse model, with sensible collapse behavior.
\end{abstract}

DOI: 10.1103/PhysRevResearch.1.033040

\section{INTRODUCTION}

The nonrelativistic continuous-spontaneous-localization (CSL) model $[1,2]$ is a response to the quantum measurement problem. That problem is a perceived deficiency in the standard quantum theory (SQT) description of a measurement where, in order to have the state vector represent the physical situation, its Schrödinger equation evolution is abandoned, to be replaced by an ill-defined "collapse of the state vector" postulate, whereafter the Schrödinger equation evolution is resumed, a procedure to which Schrödinger himself objected [3]. There are a number of other approaches to resolving this problem, prominent among which are the pilot wave theory of de Broglie-Bohm, many worlds theories, and decohering histories (the latter two often employing the decoherence formulation) [4].

Continuous spontaneous localization is a modification of standard quantum dynamics with nonlinear and stochastic features, designed to exhibit spontaneous collapse of the wave function (for a general review, see Refs. [5,6]). The spontaneous collapse happens in such a way that small systems are effectively unaffected, while large-scale superpositions of differing distributions of matter are rapidly suppressed. The dynamical structure of CSL can then describe both the unitary development of small quantum systems and the collapse

\footnotetext{
*daniel.bedingham@rhul.ac.uk

†ppearle@hamilton.edu
}

Published by the American Physical Society under the terms of the Creative Commons Attribution 4.0 International license. Further distribution of this work must maintain attribution to the author $(s)$ and the published article's title, journal citation, and DOI. which occurs during a quantum measurement. As might be expected from a modification of SQT, CSL predicts some novel behavior. That has been, and continues to be, the subject of experimental tests over a range of phenomena [6]. At present, all results are consistent with both SQT and CSL.

Nonrelativistic CSL is a specialization of a general CSL structure that describes dynamical collapse of the state vector toward an eigenstate of any set of commuting operators, termed "collapse-generating" operators. In the case of nonrelativistic CSL, these are mass-density operators at each point of space (smeared over a characteristic distance). In this paper, we take the collapse-generating operators to be the relativistic scalar quantum field operator $\hat{\phi}(x, 0)$ associated with particles of mass $m$ at each point of space.

The state vector in the Schrödinger picture and in the interaction picture are respectively

$$
\begin{aligned}
|\psi, T\rangle_{S} & =\mathcal{T} e^{-i \int_{0}^{T} d t \hat{H}-\frac{1}{4 \lambda} \int_{0}^{T} d x d t[w(x, t)-2 \lambda \hat{\phi}(x, 0)]^{2}}|\psi, 0\rangle, \\
|\psi, T\rangle_{I} & =\mathcal{T} e^{-\frac{1}{4 \lambda} \int_{0}^{T} d x d t[w(x, t)-2 \lambda \hat{\phi}(x, t)]^{2}}|\psi, 0\rangle,
\end{aligned}
$$

where $\mathcal{T}$ is the time-ordering operator, $\hat{H}$ is the Hamiltonian for the freely evolving scalar field, $w(x, t)$ is a random realvalued scalar field of white noise type, and these state vectors (whose norms are not 1) are to occur in nature with probability proportional to the state vector squared norm.

It is known [7,8] that this model generates problematic divergent energy increase and this will be demonstrated in Sec. III. Realistic collapse models generally display some energy increase as a result of the fact that localization is necessarily accompanied by some spreading in momentum. This offers the possibility of experimentally testing CSL via heating effects. Here we leave aside the issue of infinite energy increase, assuming that it can eventually be regulated in some way. Instead we focus on the collapse behavior. For an initial 
state representing a superposition of two differently located clumps of particles we will find that the state collapses toward an eigenstate of the scalar field representing either one of the two clumps of particles.

The density matrix in the Schrödinger picture satisfies the Lindblad evolution equation

$$
\frac{d}{d t} \hat{\rho}(t)=-i[\hat{H}, \hat{\rho}(t)]-\frac{\lambda}{2} \int d x[\hat{\phi}(x, 0),[\hat{\phi}(x, 0), \hat{\rho}(t)]] .
$$

We will now show how the density matrix can be constructed as the direct product of harmonic oscillator density matrices associated with each momentum mode (this is similar, but not identical to, the construction in Ref. [7]).

Write the particle annihilation operator as $\hat{a}(k) \equiv \hat{a}_{k} / \sqrt{d k}$, so $\left[\hat{a}_{k}, \hat{a}_{k^{\prime}}^{\dagger}\right]=\delta_{k, k^{\prime}}$. Next, define position and momentum operators associated with each momentum mode (of course, these have nothing to do with position and momentum for the actual particles), for $k>0, \hat{a}_{k} \equiv \frac{1}{\sqrt{2}}\left[\hat{x}_{k 1}+i \hat{p}_{k 1}\right]$ and, for $k<0, \hat{a}_{-|k|} \equiv \hat{b}_{|k|} \equiv \frac{1}{\sqrt{2}}\left[\hat{x}_{|k| 2}+i \hat{p}_{|k| 2}\right]$. Finally define center of mass and relative position and momentum operators for only $k>0, \hat{X}_{k} \equiv \frac{1}{2}\left[\hat{x}_{k 1}+\hat{x}_{k 2}\right], \hat{P}_{k} \equiv\left[\hat{p}_{k 1}+\hat{p}_{k 2}\right], \hat{x}_{k} \equiv\left[\hat{x}_{k 1}-\hat{x}_{k 2}\right], \hat{p}_{k} \equiv \frac{1}{2}\left[\hat{p}_{k 1}-\hat{p}_{k 2}\right]$.

The scalar field $\hat{\phi}(x, 0)=\int_{-\infty}^{\infty} d k \sqrt{\frac{1}{4 \pi \omega(k)}}\left[\hat{a}(k) e^{i k x}+\hat{a}^{\dagger}(k) e^{-i k x}\right]$ may then be written as

$$
\begin{aligned}
\hat{\phi}(x, 0) & =\int_{0}^{\infty} d k \sqrt{\frac{1}{4 \pi \omega(k)}}\left\{\left[\hat{a}(k)+\hat{b}^{\dagger}(k)\right] e^{i k x}+\left[\hat{a}^{\dagger}(k)+\hat{b}(k)\right] e^{-i k x}\right\} \\
& =\sum_{k>0} \sqrt{\frac{d k}{2 \pi \omega(k)}}\left[\left(\hat{X}_{k}+i \hat{p}_{k}\right) e^{i k x}+\left(\hat{X}_{k}-i \hat{p}_{k}\right) e^{-i k x}\right] .
\end{aligned}
$$

Define eigenvectors of the center of mass position and relative momentum operators, $|X\rangle|p\rangle$, where $\hat{X}_{k}|X\rangle|p\rangle=$ $X_{k}|X\rangle|p\rangle, \hat{p}_{k}|X\rangle|p\rangle=p_{k}|X\rangle|p\rangle$, so $|X\rangle|p\rangle=\prod_{k>0}\left|X_{k}\right\rangle\left|p_{k}\right\rangle$, and $\left\langle X_{k^{\prime}}^{\prime} \mid X_{k}\right\rangle=\delta_{k k^{\prime}} \delta\left(X_{k}^{\prime}-X_{k}\right),\left\langle p_{k^{\prime}}^{\prime} \mid p_{k}\right\rangle=\delta_{k k^{\prime}} \delta\left(p_{k}^{\prime}-p_{k}\right)$.

Then, since $\left[\hat{\phi}(x, 0), \hat{\phi}\left(x^{\prime}, 0\right)\right]=0$, there is a joint eigenvector (all $x$ ) satisfying $\hat{\phi}(x, 0)|f\rangle \equiv f(x)|f\rangle$, with real arbitrary eigenvalue functions $f(x)=\frac{1}{\sqrt{2 \pi}} \int_{-\infty}^{\infty} d k \tilde{f}(k) e^{i k x}$ [where $\left.\tilde{f}^{*}(k)=\tilde{f}(-k)\right]$. It follows from $(1.3)$ that $|X\rangle|p\rangle$ is an eigenstate of $\hat{\phi}(x, 0)$

$$
\hat{\phi}(x, 0)|X\rangle|p\rangle=\sum_{k>0} \sqrt{\frac{d k}{2 \pi \omega(k)}}\left[\left(X_{k}+i p_{k}\right) e^{i k x}+\left(X_{k}-i p_{k}\right) e^{-i k x}\right]|X\rangle|p\rangle,
$$

so, with $\tilde{f}(k)=\tilde{f}_{R}(k)+i \tilde{f}_{I}(k)$, we identify $\frac{1}{\sqrt{d k \omega(k)}} X_{k}=\tilde{f}_{R}(k), \frac{1}{\sqrt{d k \omega(k)}} p_{k}=\tilde{f}_{I}(k)$, and then

$$
|f\rangle=\prod_{k>0}\left|\sqrt{\omega(k) d k} \tilde{f}_{R}(k)\right\rangle\left|\sqrt{\omega d k} \tilde{f}_{I}(k)\right\rangle \equiv \prod_{k>0}|f\rangle_{k} .
$$

According to $(1.5),{ }_{k}\left\langle f^{\prime} \mid f\right\rangle_{k}=\frac{1}{\omega(k) d k} \delta\left[f_{R}^{\prime}(k)-f_{R}(k)\right] \delta\left[f_{I}^{\prime}(k)-f_{I}(k)\right]$.

By inserting (1.3) into (1.2), the Lindblad equation becomes

$$
\frac{d}{d t} \hat{\rho}(t)=\sum_{k>0}\left\{-i \omega(k)\left[\hat{X}_{k}^{2}+\frac{1}{4} \hat{P}_{k}^{2}, \hat{\rho}(t)\right]-\frac{\lambda}{\omega(k)}\left[\hat{X}_{k},\left[\hat{X}_{k}, \hat{\rho}(t)\right]\right]-i \omega(k)\left[\frac{1}{4} \hat{x}_{k}^{2}+\hat{p}_{k}^{2}, \hat{\rho}(t)\right]-\frac{\lambda}{\omega(k)}\left[\hat{p}_{k},\left[\hat{p}_{k}, \hat{\rho}(t)\right]\right]\right\} .
$$

The problem can be reduced to solving individual mode equations if the initial density matrix can be written as $\hat{\rho}(0)=$ $\sum_{v} c_{v} \prod_{k} \hat{\rho}_{v, k}(0)$. This defines a state whose modes are separable. For initial states of this type the density matrix at later times may be written as $\hat{\rho}(t)=\sum_{\nu} c_{\nu} \prod_{k} \hat{\rho}_{\nu, k}(t)$ where

$$
\frac{d}{d t} \hat{\rho}_{v, k}(t)=-i \omega(k)\left[\hat{X}_{k}^{2}+\frac{1}{4} \hat{P}_{k}^{2}, \hat{\rho}_{v, k}(t)\right]-\frac{\lambda}{\omega(k)}\left[\hat{X}_{k},\left[\hat{X}_{k}, \hat{\rho}_{v, k}(t)\right]\right]-i \omega(k)\left[\frac{1}{4} \hat{x}_{k}^{2}+\hat{p}_{k}^{2}, \hat{\rho}_{v, k}(t)\right]-\frac{\lambda}{\omega(k)}\left[\hat{p}_{k},\left[\hat{p}_{k}, \hat{\rho}_{v, k}(t)\right]\right],
$$

subject to the initial condition $\hat{\rho}_{v, k}(0)$.

\section{INITIAL STATE}

We will be considering the initial state

$$
\begin{aligned}
|\psi, 0\rangle & =\frac{1}{\sqrt{2\left[1+\left\langle\ell_{1} \mid \ell_{2}\right\rangle\right]}}\left[\left|\ell_{1}\right\rangle+\left|\ell_{2}\right\rangle\right], \\
\hat{\rho}(0) & =\frac{1}{2\left[1+\left\langle\ell_{1} \mid \ell_{2}\right\rangle\right]}\left[\left|\ell_{1}\right\rangle+\left|\ell_{2}\right\rangle\right]\left[\left\langle\ell_{1}\right|+\left\langle\ell_{2}\right|\right],
\end{aligned}
$$


where $\left|\ell_{1}\right\rangle,\left|\ell_{2}\right\rangle$ represent two clumps of particles at widely separated locations:

$$
|\ell\rangle \equiv e^{\int_{-\infty}^{\infty} d k \hat{a}^{\dagger}(k) \tilde{\chi}(k)}|0\rangle e^{-\frac{1}{2} \int_{-\infty}^{\infty} d k|\tilde{\chi}(k)|^{2}}=\prod_{k>0} e^{\sqrt{2 d k}\left[\left(\hat{X}_{k}-i \frac{1}{2} \hat{P}_{k}\right) \tilde{\chi}_{R}(k)+\left(\hat{p}_{k}+i \frac{1}{2} \hat{x}_{k}\right) \tilde{x}_{l}(k)\right]}|0\rangle_{k} e^{-d k|\tilde{\chi}(k)|^{2}} \equiv \prod_{k>0}|\ell\rangle_{k},
$$

with

$$
\begin{aligned}
& \chi(x) \equiv N^{1 / 2} \frac{1}{\left(2 \pi \sigma^{2}\right)^{1 / 4}} e^{-\frac{1}{4 \sigma^{2}}(x-\ell)^{2}}=\frac{1}{\sqrt{2 \pi}} \int_{-\infty}^{\infty} d k \tilde{\chi}(k) e^{i k x}, \\
& \tilde{\chi}(k)=N^{1 / 2}\left(\frac{2 \sigma^{2}}{\pi}\right)^{1 / 4} e^{-k^{2} \sigma^{2}} e^{-i k \ell},
\end{aligned}
$$

and $|0\rangle=\prod_{k>0}|0\rangle_{k}$ is the vacuum (no particle) state, i.e., $|l\rangle$ is a form of coherent state.

The particle number density operator is $\hat{\xi}^{\dagger}(x) \hat{\xi}(x)$ [where $\hat{\xi}(x)=\frac{1}{\sqrt{2 \pi}} \int_{-\infty}^{\infty} d k \hat{a}(k) e^{i k x}$ ]. Since $\left\langle\ell\left|\hat{\xi}^{\dagger}(x) \hat{\xi}(x)\right| \ell\right\rangle=\chi^{2}(x)$, this state may be thought of as representing $\approx N$ particles (the particle number operator $\hat{N}=\int_{-\infty}^{\infty} d x \hat{\xi}^{\dagger}(x) \hat{\xi}(x)$ has mean value $N$ and mean-squared value $N^{2}+N$, so the standard deviation of the number of particles divided by $N$ is $1 / \sqrt{N}$ ) centered at $\ell$ and spread over width $\approx \sigma \ll\left|\ell_{1}-\ell_{2}\right|$.

The states $\left|\ell_{1}\right\rangle,\left|\ell_{2}\right\rangle$ are not quite orthogonal:

$$
\left\langle\ell_{1} \mid \ell_{2}\right\rangle=e^{\int_{-\infty}^{\infty} d k \tilde{\chi}_{1}^{*}(k) \tilde{x}_{2}(k)} e^{-\frac{1}{2} \int_{-\infty}^{\infty} d k\left|\tilde{\chi}_{1}(k)\right|^{2}} e^{-\frac{1}{2} \int_{-\infty}^{\infty} d k\left|\tilde{\chi}_{2}(k)\right|^{2}}=e^{-N\left[1-e^{-\frac{\left(\ell_{1}-\ell_{2}\right)^{2}}{8 \sigma^{2}}}\right]} .
$$

However, we will assume the number of particles $N$ is so large that we may neglect $e^{-N}$ compared to 1 , in Secs. IV and V. Thus, we will take the state vector normalization factor in Eq. (2.1) to be simply $\frac{1}{\sqrt{2}}$. Therefore, we have to solve Eq. (1.7) for

$$
\hat{\rho}_{11 k}(t), \hat{\rho}_{12 k}(t), \hat{\rho}_{21 k}(t), \hat{\rho}_{22 k}(t) \text {, with corresponding initial conditions } \hat{\rho}_{s s^{\prime} k}(0)=\left|\ell_{s}\right\rangle_{k k}\left\langle\ell_{s^{\prime}}\right| \text {. }
$$

We will need to know $|0\rangle_{k}$ and $|\ell\rangle_{k}$ in the $\left|X_{k}\right\rangle\left|p_{k}\right\rangle$ basis.

For $|0\rangle_{k}$, since $\left(\hat{X}_{k}+i \frac{1}{2} \hat{P}_{k}\right)|0\rangle_{k}=0,\left(\hat{p}_{k}-i \frac{1}{2} \hat{x}_{k}\right)|0\rangle_{k}=0$ :

$$
\left\langle X_{k}\right|\left\langle p_{k} \mid 0\right\rangle_{k}=\sqrt{\frac{2}{\pi}} e^{-X_{k}^{2}} e^{-p_{k}^{2}}
$$

For $|\ell\rangle_{k}$, we apply the Campbell-Baker-Hausdorff theorem to see that

$$
e^{\alpha\left(X-\frac{1}{2} \frac{d}{d X}\right)} e^{-X^{2}} e^{-\frac{1}{2} \alpha^{2}}=e^{\alpha X} e^{-\alpha \frac{1}{2} \frac{d}{d X}} e^{-X^{2}} e^{-\frac{3}{4} \alpha^{2}}=e^{\alpha X} e^{-\left(X-\frac{1}{2} \alpha\right)^{2}} e^{-\frac{3}{4} \alpha^{2}}=e^{-(X-\alpha)^{2}} .
$$

Therefore, it follows from (2.2) and (2.7) that

$$
\left\langle X_{k}\right|\left\langle p_{k} \mid \ell\right\rangle_{k}=\sqrt{\frac{2}{\pi}} e^{-\left[X_{k}-\sqrt{2 d k} \tilde{\chi}_{R}(k)\right]^{2}} e^{-\left[p_{k}-\sqrt{2 d k} \tilde{\chi}_{l}(k)\right]^{2}},
$$

or, using (1.5),

$$
{ }_{k}\langle f \mid \ell\rangle_{k}=\sqrt{\frac{2}{\pi}} e^{-d k\left[\sqrt{\omega(k)} \tilde{f}_{R}(k)-\sqrt{2} \tilde{\chi}_{R}(k)\right]^{2}} e^{-d k\left[\sqrt{\omega(k)} \tilde{f}_{l}(k)-\sqrt{2} \tilde{\chi}_{l}(k)\right]^{2}} \approx \sqrt{\frac{2}{\pi}} e^{-d k \omega(k)\left[\tilde{f}_{R}(k)-\sqrt{\frac{2}{m}} \tilde{\chi}_{R}(k)\right]^{2}} e^{-d k \omega(k)\left[\tilde{f}_{l}(k)-\sqrt{\frac{2}{m}} \tilde{\chi}_{l}(k)\right]^{2}},
$$

where we have made the approximation $\frac{1}{\sqrt{\omega(k)}} \approx \frac{1}{\sqrt{m}}$ since $\tilde{\chi}(k)$ is non-negligible only for $k<<m$.

\section{PARTICLE PRODUCTION}

The eigenstates of the scalar field $|f\rangle$ are superpositions of states with all numbers of particles. To see this, we note that an alternative form for $|f\rangle$ is

$$
|f\rangle \sim e^{-\int_{0}^{\infty} d k \hat{a}^{\dagger}(k) \hat{b}^{\dagger}(k)} e^{\int_{0}^{\infty} d k \sqrt{2 \omega}\left[\hat{a}^{\dagger}(k) \tilde{f}(k)+\hat{b}^{\dagger}(k) \tilde{f}^{*}(k)\right]}|0\rangle \sim e^{-\sum_{k>0} \hat{a}_{k}^{\dagger} \hat{b}_{k}^{\dagger}} e^{\sum_{k>0} \sqrt{2 \omega d k}\left[\hat{a}_{k}^{\dagger} \tilde{f}(k)+\hat{b}_{k}^{\dagger} \tilde{f}^{*}(k)\right]}|0\rangle .
$$

This is because the right side of (3.1) is an eigenstate of $\hat{\phi}(x, 0)$ with eigenvalue $f(x)$ :

$$
\begin{aligned}
& \hat{\phi}(x, 0) e^{-\sum_{k>0} \hat{a}_{k}^{\dagger} \hat{b}_{k}^{\dagger}} e^{\left.\sum_{k>0} \sqrt{2 \omega d k} \hat{a}_{k}^{\dagger} \tilde{f}(k)+\hat{b}_{k}^{\dagger} \tilde{f}^{*}(k)\right]}|0\rangle \\
& =\sum_{k>0} \sqrt{\frac{d k}{4 \pi \omega(k)}}\left[\left(\hat{a}_{k}+\hat{b}_{k}^{\dagger}\right) e^{i k x}+\left(\hat{a}_{k}^{\dagger}+\hat{b}_{k}\right) e^{-i k x}\right] e^{-\sum_{k>0} \hat{a}_{k}^{\dagger} \hat{k}_{k}^{\dagger} e^{\sum_{k>0} \sqrt{2 \omega d k}\left(\hat{a}_{k}^{\dagger} \tilde{f}(k)+\hat{b}_{k}^{\dagger} \tilde{f}^{*}(k)\right]}|0\rangle} \\
& =e^{-\sum_{k>0} \hat{a}_{k}^{\dagger} \hat{b}_{k}^{\dagger}} \sum_{k>0} \sqrt{\frac{d k}{4 \pi \omega(k)}}\left[\hat{a}_{k} e^{i k x}+\hat{b}_{k} e^{-i k x}\right] e^{\left.\sum_{k>0} \sqrt{2 \omega d k} \hat{a}_{k}^{\dagger} \tilde{f}(k)+\hat{b}_{k}^{\dagger} \tilde{f}^{*}(k)\right]}|0\rangle
\end{aligned}
$$




$$
\begin{aligned}
& =e^{-\sum_{k>0} \hat{a}_{k}^{\dagger} \hat{b}_{k}^{\dagger}} e^{\sum_{k>0} \sqrt{2 \omega d k}\left[\hat{a}_{k}^{\dagger} \tilde{f}(k)+\hat{b}_{k}^{\dagger} \tilde{f}^{*}(k)\right]}|0\rangle \sum_{k>0} d k \sqrt{\frac{1}{2 \pi}}\left[\tilde{f}_{R}(k) e^{i k x}+\tilde{f}_{I}(k) e^{-i k x}\right] \\
& =e^{-\sum_{k>0} \hat{a}_{k}^{\dagger} \hat{b}_{k}^{\dagger}} e^{\left.\sum_{k>0} \sqrt{2 \omega d k} \hat{a}_{k}^{\dagger} \tilde{f}(k)+\hat{b}_{k}^{\dagger} \tilde{f}^{*}(k)\right]}|0\rangle f(x) .
\end{aligned}
$$

For example, the state $|f=0\rangle$ may be written as

$$
|f=0\rangle \sim e^{-\sum_{k>0} \hat{a}_{k}^{\dagger} b_{k}^{\dagger}}|0\rangle=\prod_{k} \sum_{n=0}^{\infty}(-1)^{n} \frac{1}{n !}\left(\hat{a}_{k}^{\dagger} \hat{b}_{k}^{\dagger}\right)^{n}|0\rangle_{k}=\prod_{k} \sum_{n=0}^{\infty}(-1)^{n}|n\rangle_{k}|n\rangle_{-k} .
$$

For $f(x) \neq 0$, this is multiplied by a factor which puts even more particles into each mode.

Since any eigenstate of the scalar field has infinite particles, and the collapse end product is one or another of these eigenstates, the collapse process must generate these particles. We calculate the rate of particle production (this is well known [1], but repeated here for completeness, in the formalism of this paper). It follows from (1.6) that

$$
\frac{d}{d t} \overline{\hat{O}} \equiv \frac{d}{d t} \operatorname{Tr} \hat{O} \hat{\rho}(t)=\sum_{k>0}\left\{i \omega(k) \operatorname{Tr} \hat{\rho}(t)\left[\hat{a}_{k}^{\dagger} \hat{a}_{k}+\hat{b}_{k}^{\dagger} \hat{b}_{k}, \hat{O}\right]-\frac{\lambda}{\omega(k)} \operatorname{Tr} \hat{\rho}(t)\left[\hat{X}_{k},\left[\hat{X}_{k}, \hat{O}\right]\right]-\frac{\lambda}{\omega(k)} \operatorname{Tr} \hat{\rho}(t)\left[\hat{p}_{k},\left[\hat{p}_{k}, \hat{O}\right]\right]\right\},
$$

where $\operatorname{Tr}$ is the trace operation. Setting $\hat{O}$ equal to

$$
\hat{a}_{k}^{\dagger} \hat{a}_{k}+\hat{b}_{k}^{\dagger} \hat{b}_{k}=\hat{X}_{k}^{2}+\frac{1}{4} \hat{P}_{k}^{2}+\hat{p}_{k}^{2}+\frac{1}{4} \hat{x}_{k}^{2}-1 \text { and } \hat{a}_{k}^{\dagger} \hat{a}_{k}-\hat{b}_{k}^{\dagger} \hat{b}_{k}=\hat{X}_{k} \hat{x}_{k}+\hat{P}_{k} \hat{p}_{k},
$$

and inserting this into (3.4), we find $\frac{d}{d t}\left[\overline{\hat{a}_{k}^{\dagger} \hat{a}_{k}+\hat{b}_{k}^{\dagger} \hat{b}_{k}}\right]=\frac{\lambda}{\omega(k)}, \frac{d}{d t}\left[\overline{\hat{a}_{k}^{\dagger} \hat{a}_{k}-\hat{b}_{k}^{\dagger} \hat{b}_{k}}\right]=0$ and so

$$
\overline{\hat{a}_{k}^{\dagger} \hat{a}_{k}(t)}=\frac{\lambda t}{2 \omega(k)}+\overline{\hat{a}_{k}^{\dagger} \hat{a}_{k}(0)}, \quad \overline{\hat{b}_{k}^{\dagger} \hat{b}_{k}(t)}=\frac{\lambda t}{2 \omega(k)}+\overline{\hat{b}_{k}^{\dagger} \hat{b}_{k}(0)} .
$$

In particular, each momentum mode contributes the same rate of energy increase,

$$
\overline{\hat{H}_{k}(t)}=\omega(k)\left[\overline{\hat{a}_{k}^{\dagger} \hat{a}_{k}+\hat{b}_{k}^{\dagger} \hat{b}_{k}}\right]=\lambda t+\overline{\hat{H}_{k}(0)} .
$$

To clarify what that means, we use $d k=2 \pi / \int d x$, which follows from

$$
\left[\hat{a}_{k}, \hat{a}_{k}^{\dagger}\right]=1=d k\left[\hat{a}(k), \hat{a}^{\dagger}(k)\right]=d k \delta(k-k)=d k \frac{1}{2 \pi} \int d x e^{i x(k-k)}=d k \frac{1}{2 \pi} \int d x .
$$

Replacing $\int d x$ by the length $\equiv L$, and inserting this into (3.7):

$$
\frac{\overline{\hat{H}_{k}(t)}}{L}=\frac{d k}{2 \pi} \lambda t+\frac{\overline{\hat{H}_{k}(0)}}{L} .
$$

That is, the energy per unit length contributed by each mode of $\mid$ momentum $\mid=k>0$, of width $d k$, grows linearly with time. The net contribution of a finite range of modes is finite, but the net contribution from all modes is infinite at any finite time. And, at infinite time, each mode has infinite energy/length, which conforms with the end collapse to field eigenstates which have an infinite number of particles in each mode.

\section{COLLAPSE OF A SUPERPOSITION WHEN $\boldsymbol{H}=0$}

The initial density matrix is to be $\left.\hat{\rho}(0)=\frac{1}{2}\left[\left|\ell_{1}\right\rangle+\ell_{2}\right\rangle\right]\left[\left\langle\ell_{1}\right|+\left\langle\ell_{2}\right|\right]$, corresponding to a superposition of $\approx N$ particles in two widely separated clumps,

When $H=0$, the solution of the Lindblad equation (1.7), in the $\left|X_{k}\right\rangle\left|p_{k}\right\rangle$ basis and in the $|f\rangle_{k}$ basis is, using (2.8) and (2.9),

$$
\begin{aligned}
\left\langle X_{k}\left|\left\langle p_{k}\left|\hat{\rho}_{s s^{\prime} k}(t)\right| X_{k}^{\prime}\right\rangle\right| p_{k}^{\prime}\right\rangle & =e^{-\frac{\lambda t}{\omega(k)}\left\{\left[X_{k}-X_{k}^{\prime}\right]^{2}+\left[p_{k}-p_{k}^{\prime}\right]^{2}\right\}} \frac{2}{\pi} e^{-\left[X_{k}-\sqrt{2 d k} \tilde{\chi}_{R s}(k)\right]^{2}-\left[p_{k}-\sqrt{2 d k} \tilde{\chi}_{l s}(k)\right]^{2}} e^{-\left[X_{k}^{\prime}-\sqrt{2 d k} \tilde{\chi}_{R s^{\prime}}(k)\right]^{2}-\left[p_{k}^{\prime}-\sqrt{2 d k} \tilde{X}_{s^{\prime}}(k)\right]^{2}}, \\
{ }_{k}\left\langle f\left|\hat{\rho}_{s s^{\prime} k}(t)\right| f^{\prime}\right\rangle_{k} & =e^{-\lambda t d k\left|\tilde{f}(k)-\tilde{f}^{\prime}(k)\right|^{2}} \frac{2}{\pi} e^{-d k \omega(k)\left|\tilde{f}(k)-\sqrt{\frac{2}{m}} \tilde{\chi}_{s}(k)\right|^{2}} e^{-d k \omega(k)\left|\tilde{f}^{\prime}(k)-\sqrt{\frac{2}{m}} \tilde{\chi}_{s^{\prime}}(k)\right|^{2}} .
\end{aligned}
$$

Using (4.1), we then can construct the complete density matrix in the $|f\rangle$ basis:

$$
\begin{aligned}
\left\langle f|\hat{\rho}(t)| f^{\prime}\right\rangle & =\frac{1}{2} e^{-\lambda t \int_{0}^{\infty} d k\left|\tilde{f}(k)-\tilde{f}^{\prime}(k)\right|^{2}} \sum_{s s^{\prime}=1}^{2} e^{-\int_{0}^{\infty} d k \omega(k)\left|\tilde{f}(k)-\sqrt{\frac{2}{m}} \tilde{x}_{s}(k)\right|^{2}} e^{-\int_{0}^{\infty} d k \omega(k)\left|\tilde{f}^{\prime}(k)-\sqrt{\frac{2}{m}} \tilde{x}_{s^{\prime}}(k)\right|^{2}} \\
& =\frac{1}{2} e^{-\lambda t \frac{1}{2} \int_{-\infty}^{\infty} d x\left[f(x)-f^{\prime}(x)\right]^{2}} \sum_{s s^{\prime}=1}^{2} e^{-\frac{1}{2} \int_{-\infty}^{\infty} d x\left\{\left[m^{2}-d^{2} / d x^{2}\right]^{1 / 4}\left[f(x)-\sqrt{\frac{2}{m}} x_{s}(x)\right]\right\}^{2}} e^{-\frac{1}{2} \int_{-\infty}^{\infty} d x\left\{\left[m^{2}-d^{2} / d x^{2}\right]^{1 / 4}\left[f^{\prime}(x)-\sqrt{\frac{2}{m}} x_{s^{\prime}}(x)\right]\right\}^{2}},
\end{aligned}
$$

using Parseval's theorem, and absorbing the factors $\frac{2}{\pi}$ in the functional integral element $D f \equiv \prod_{k>0} d \tilde{f}_{R}(k) d \tilde{f}_{I}(k) \frac{2 d k \omega(k)}{\pi}$. 
The first exponential in Eqs. (4.2) is largest when $f(x) \approx f^{\prime}(x)$ : this term is responsible for the ultimate $(t \rightarrow \infty)$ collapse to eigenstates of the scalar field.

The exponential terms in the sum are largest when $f(x) \approx \sqrt{2 / m} \chi_{s}(x), f^{\prime}(x) \approx \sqrt{2 / m} \chi_{s^{\prime}}(x)$. For the contribution to the density matrix of the diagonal elements of the initial density matrix, $s=s^{\prime}$, this is compatible with $f(x)-f^{\prime}(x) \approx 0$, and so there can be relatively little decay.

However, for the terms associated with the off-diagonal elements of the initial density matrix, $s \neq s^{\prime}$, the conditions $f(x) \approx f^{\prime}(x), f(x) \approx \sqrt{2 / m} \chi_{s}(x), f^{\prime}(x) \approx \sqrt{2 / m} \chi_{s^{\prime}}(x)$ cannot all be satisfied, and so these terms exponentially decay as time progresses.

To see this in more detail we can find the values of $\tilde{f}(k)$ and $\tilde{f}^{\prime}(k)$ which maximize the exponent in Eq. (4.1). These are

$$
\begin{aligned}
& \tilde{f}_{0}(k)=\frac{1}{2 \lambda t+\omega} \sqrt{\frac{2}{m}}\left\{\lambda t\left[\tilde{\chi}_{s}(k)+\tilde{\chi}_{s^{\prime}}(k)\right]+\omega \tilde{\chi}_{s}(k)\right\}, \\
& \tilde{f}_{0}^{\prime}(k)=\frac{1}{2 \lambda t+\omega} \sqrt{\frac{2}{m}}\left\{\lambda t\left[\tilde{\chi}_{s}(k)+\tilde{\chi}_{s^{\prime}}(k)\right]+\omega \tilde{\chi}_{s^{\prime}}(k)\right\} .
\end{aligned}
$$

When $\tilde{f}$ and $\tilde{f}^{\prime}$ take these forms, the exponent in Eq. (4.1) takes the value

$$
-\frac{2 \lambda t \omega}{m(2 \lambda t+\omega)}\left|\tilde{\chi}_{s}(k)-\tilde{\chi}_{s^{\prime}}(k)\right|^{2} .
$$

Therefore, in the limit that $t \rightarrow \infty$, if $s=s^{\prime}$, the exponent is a maximum with value 0 when $\tilde{f}(k)=\tilde{f}^{\prime}(k)=\sqrt{2 / m} \tilde{\chi}_{s}(k)$; and, if $s \neq s^{\prime}$, the exponent is a maximum with value $-(\omega / m)\left|\tilde{\chi}_{s}-\tilde{\chi}_{s^{\prime}}\right|^{2} \approx-\left|\tilde{\chi}_{s}-\tilde{\chi}_{s^{\prime}}\right|^{2}$ when $\tilde{f}(k)=\tilde{f}^{\prime}(k)=\sqrt{1 / 2 m}\left[\tilde{\chi}_{s}(k)+\tilde{\chi}_{s^{\prime}}(k)\right]$. In the latter case, taking into account all modes,

$$
\lim _{t \rightarrow \infty}\left\langle f_{0}\left|\rho_{s s^{\prime}}\right| f_{0}^{\prime}\right\rangle=e^{-\int_{0}^{\infty} d k\left|\tilde{\chi}_{s}-\tilde{\chi}_{s^{\prime}}\right|^{2}}=e^{-N\left[1-e^{-\frac{\left(\ell_{1}-\ell_{2}\right)^{2}}{8 \sigma^{2}}}\right]}
$$

where we have used (2.4). This represents the overlap between states $\left|l_{1}\right\rangle$ and $\left|l_{2}\right\rangle$ which for large $N$ is negligibly small. This reflects the small probability at large $t$ of the particles belonging to both clumps.

In nonrelativistic CSL the collapse, in a superposition of two clumps of matter, toward one or another clump occurs because the clumps represent two quite different mass-density distributions. Here, the collapse occurs because the two clumps correspond to two quite different scalar field distributions which are approximately proportional to the two separated clump wave functions representing two quite different mass-density distributions.

Last, let's look at the density matrix's components in the initial clump state basis. Using (2.8) and (4.1),

$$
\begin{aligned}
{ }_{k}\left\langle\ell_{i}\left|\hat{\rho}_{s s^{\prime} k}(t)\right| \ell_{j}\right\rangle_{k}= & \int d X_{k} d p_{k} \int d X_{k}^{\prime} d p_{k}^{\prime} e^{-\frac{\lambda t}{\omega(k)}\left\{\left[X_{k}-X_{k}^{\prime}\right]^{2}+\left[p_{k}-p_{k}^{\prime}\right]^{2}\right\}}{ }_{k}\left\langle\ell_{i} \mid X_{k}\right\rangle\left|p_{k}\right\rangle\left\langle X_{k}\left|\left\langle p_{k} \mid \ell_{s}\right\rangle_{k k}\left\langle\ell_{s^{\prime}} \mid X_{k}^{\prime}\right\rangle\right| p_{k}^{\prime}\right\rangle\left\langle X_{k}^{\prime}\right|\left\langle p_{k}^{\prime} \mid \ell_{j}\right\rangle_{k} \\
= & \int d X_{k} d p_{k} \int d X_{k}^{\prime} d p_{k}^{\prime} e^{-\frac{\lambda t}{\omega(k)}\left\{\left[X_{k}-X_{k}^{\prime}\right]^{2}+\left[p_{k}-p_{k}^{\prime}\right]^{2}\right\}} \\
& \times \sqrt{\frac{2}{\pi}} e^{-\left[X_{k}-\sqrt{2 d k} \tilde{\chi}_{i R}(k)\right]^{2}} e^{-\left[p_{k}-\sqrt{2 d k} \tilde{\chi}_{i I}(k)\right]^{2}} \sqrt{\frac{2}{\pi}} e^{-\left[X_{k}-\sqrt{2 d k} \tilde{\chi}_{s R}(k)\right]^{2}} e^{-\left[p_{k}-\sqrt{2 d k} \tilde{\chi}_{s I}(k)\right]^{2}} \\
& \times \sqrt{\frac{2}{\pi}} e^{-\left[X_{k}^{\prime}-\sqrt{2 d k} \tilde{\chi}_{j R}(k)\right]^{2}} e^{-\left[p_{k}^{\prime}-\sqrt{2 d k} \tilde{\chi}_{j I}(k)\right]^{2}} \sqrt{\frac{2}{\pi}} e^{-\left[X_{k}^{\prime}-\sqrt{2 d k} \tilde{x}_{s^{\prime} R}(k)\right]^{2}} e^{-\left[p_{k}^{\prime}-\sqrt{2 d k} \tilde{\chi}_{s^{\prime} I}(k)\right]^{2}} .
\end{aligned}
$$

We evaluate the integrals in Eq. (4.6) using

$$
\int_{-\infty}^{\infty} d x d x^{\prime} e^{-\alpha\left(x-x^{\prime}\right)^{2}} e^{-(x-A)^{2}} e^{-(x-B)^{2}} e^{-\left(x^{\prime}-C\right)^{2}} e^{-\left(x^{\prime}-D\right)^{2}}=\frac{\pi}{2 \sqrt{\alpha+1}} e^{-\frac{1}{2}(A-B)^{2}} e^{-\frac{1}{2}(C-D)^{2}} e^{-\frac{\alpha(A+B-C-D)^{2}}{4(\alpha+1)}},
$$

obtaining

$$
\begin{aligned}
{ }_{k}\left\langle\ell_{i}\left|\hat{\rho}_{s s^{\prime} k}(t)\right| \ell_{j}\right\rangle_{k} & =\frac{1}{\frac{\lambda t}{\omega(k)}+1} e^{-\frac{\frac{\lambda t}{\omega(k)}}{2\left[1+\frac{\lambda t}{\omega(k)} d k\left|\tilde{\chi}_{i}(k)+\tilde{\chi}_{s}(k)-\tilde{\chi}_{j}(k)-\tilde{\chi}_{s^{\prime}}(k)\right|^{2}\right.} e^{-d k\left|\tilde{\chi}_{i}(k)-\tilde{\chi}_{s}(k)\right|^{2}} e^{-d k\left|\tilde{\chi}_{j}(k)-\tilde{\chi}_{s^{\prime}}(k)\right|^{2}},} \\
\left\langle\ell_{i}\left|\hat{\rho}_{s s^{\prime}}(t)\right| \ell_{j}\right\rangle & =K e^{-\frac{\frac{\lambda t}{m}}{2\left[1+\frac{\lambda t}{m}\right]} \int_{0}^{\infty} d k\left|\tilde{\chi}_{i}(k)+\tilde{\chi}_{s}(k)-\tilde{\chi}_{j}(k)-\tilde{\chi}_{s^{\prime}}(k)\right|^{2}} e^{-\int_{0}^{\infty} d k\left|\tilde{\chi}_{i}(k)-\tilde{\chi}_{s}(k)\right|^{2}} e^{-\int_{0}^{\infty} d k\left|\tilde{\chi}_{j}(k)-\tilde{\chi}_{s^{\prime}}(k)\right|^{2}} \\
& =K e^{-\frac{\lambda t}{4\left[1+\frac{\lambda t}{m}\right]} \int_{-\infty}^{\infty} d x\left[\chi_{i}(x)+\chi_{s}(x)-\chi_{j}(x)-\chi_{s^{\prime}}(x)\right]^{2}} e^{-\frac{1}{2} \int_{-\infty}^{\infty} d x\left[\chi_{i}(x)-\chi_{s}(x)\right]^{2}} e^{-\frac{1}{2} \int_{-\infty}^{\infty} d x\left[\chi_{j}(x)-\chi_{s^{\prime}}(x)\right]^{2}},
\end{aligned}
$$

where $K \equiv \prod_{k>0} \frac{1}{\frac{\lambda t}{\omega(k)}+1}$ [and, as mentioned earlier, replacing $\omega(k)$ by $m$ is a good approximation when multiplying $\left.\tilde{\chi}(k)\right]$. Using (3.8), we rewrite $K$ as

$$
K=e^{-\sum_{k>0} \ln \left[\frac{\lambda t}{\omega(k)}+1\right]}=e^{-\frac{L}{2 \pi} \int_{0}^{\infty} d k \ln \left[\frac{\lambda t}{\omega(k)}+1\right]}
$$

This will be discussed shortly. 
From (4.8), the elements of the density matrix are $\left[\operatorname{using} \int_{-\infty}^{\infty} d x \chi_{s}^{2}(x)=N, \int_{-\infty}^{\infty} d x \chi_{1}(x) \chi_{2}(x) \approx 0\right.$ which follow from $(2.3)$, and setting $\left.e^{-N} \approx 0\right]:$

$$
\begin{aligned}
& \left\langle\ell_{1}|\hat{\rho}(t)| \ell_{1}\right\rangle=\left\langle\ell_{2}|\hat{\rho}(t)| \ell_{2}\right\rangle=K \frac{1}{2}\left[1+e^{-2 N}+2 e^{-N\left[1+\frac{\frac{\lambda t}{m}}{2\left[\frac{\lambda t}{m}+1\right]}\right]}\right] \approx \frac{1}{2}, \\
& \left\langle\ell_{1}|\hat{\rho}(t)| \ell_{2}\right\rangle=\left\langle\ell_{2}|\hat{\rho}(t)| \ell_{1}\right\rangle=K \frac{1}{2}\left[e^{-2 N \frac{\frac{\lambda t}{m}}{\frac{\lambda t}{m}+1}}+e^{-2 N}+2 e^{-N\left[1+\frac{\frac{\lambda t}{m}}{2\left[\frac{\lambda t}{m}+1\right]}\right]}\right] \approx K \frac{1}{2} e^{-2 N \frac{\frac{\lambda t}{m}}{\frac{\lambda t}{m}+1}} .
\end{aligned}
$$

This is similar to nonrelativistic CSL collapse behavior, constant diagonal elements, and decaying off-diagonal elements, here with exponent $\sim-\lambda N t / m$ (although here the decay stops, but at negligible value $\sim e^{-2 N}$ ).

In addition, however, there is the numerical factor $K=\prod_{k>0} \frac{1}{\frac{\lambda t}{\omega(k)}+1}=\left\langle\ell_{1}|\hat{\rho}(t)| \ell_{1}\right\rangle+\left\langle\ell_{2}|\hat{\rho}(t)| \ell_{2}\right\rangle$, the trace of $\hat{\rho}(t)$ with respect to the initial clump states. This is less than 1 , and since the trace of $\hat{\rho}(t)$ is 1 , the trace of $\hat{\rho}(t)$ over all other states orthogonal to $\left|\ell_{1}\right\rangle,\left|\ell_{2}\right\rangle$ is $1-K$. These states are those for which the created particles are present, in addition to the initial clump states undergoing collapse behavior. As $t$ increases, these states of created particles come to dominate as, even for finite time, $K=0$. This tells us that there is 0 probability of no particles created for $t>0$.

\section{COLLAPSE OF A SUPERPOSITION WHEN $H \neq 0$}

The solution of Eq. (1.7) for $\left\langle X_{k}\left|\left\langle p_{k}\left|\hat{\rho}_{s s^{\prime} k}(t)\right| X_{k}^{\prime}\right\rangle\right| p_{k}^{\prime}\right\rangle$ is given in the Appendix, Eqs. (A12) and (A18) with suitable identification of parameters, $X \rightarrow \sqrt{\omega d k} \tilde{f}_{R}(k), p \rightarrow \sqrt{\omega d k} \tilde{f}_{I}(k), \gamma_{1} \rightarrow \sqrt{2 d k} \tilde{\chi}_{R s}(k), \gamma_{2} \rightarrow \sqrt{2 d k} \tilde{\chi}_{R s^{\prime}}(k), \gamma_{1}^{\prime} \rightarrow \sqrt{2 d k} \tilde{\chi}_{I s}(k), \gamma_{2}^{\prime} \rightarrow$ $\sqrt{2 d k} \tilde{\chi}_{I s^{\prime}}(k)$ :

$$
{ }_{k}\left\langle f\left|\hat{\rho}_{s s^{\prime} k}(t)\right| f^{\prime}\right\rangle_{k}=\frac{2[1-S]}{\pi[1+S]} e^{-\frac{2 S d k \omega}{\left(1-S^{2}\right)}\left|\tilde{f}(k)-\tilde{f}^{\prime}(k)\right|^{2}} e^{-d k \omega \frac{(1-S)}{(1+S)}\left\{\left|\tilde{f}(k)-\sqrt{\frac{2}{m} \frac{\tilde{\chi} s(k)-S \tilde{\chi}_{s^{\prime}}(k)}{1-S}}\right|^{2}+\left|\tilde{f}^{\prime}(k)-\sqrt{\frac{2}{m} \frac{\tilde{x}_{s^{\prime}}(k)-S \tilde{\chi}_{s}(k)}{1-S}}\right|^{2}\right\}} e^{d k 2 \frac{S}{1-S}\left|\tilde{\chi}_{s}(k)-\tilde{\chi}_{s^{\prime}}(k)\right|^{2}},
$$

with $S=\frac{\alpha}{1+\alpha}$ and $\alpha=\frac{\lambda t}{2 \omega(k)}$, so $S$ is a function of $k$.

Equation (5.1) was derived as a good approximation for $t \gg \frac{\hbar}{m c^{2}}$ which, for a neutron, is $\approx 10^{-23} \mathrm{~s}$, a negligible time on the scale of the collapse. This removed some oscillating terms. The remainder of the oscillating terms were of the form $\tilde{\chi}_{s}(k) e^{ \pm i \omega t} \approx$ $\tilde{\chi}_{s}(k) e^{ \pm i m t}$ [since $\tilde{\chi}_{s}(k)$ is of negligible amplitude for relativistic $k$ values]. Equation (5.1) holds periodically, at the closely spaced times which are integer multiples of the period, so $e^{ \pm i m t}=1$ removes the remaining oscillating terms.

Using (5.1), we then can construct the complete density matrix in the $|f\rangle$ basis:

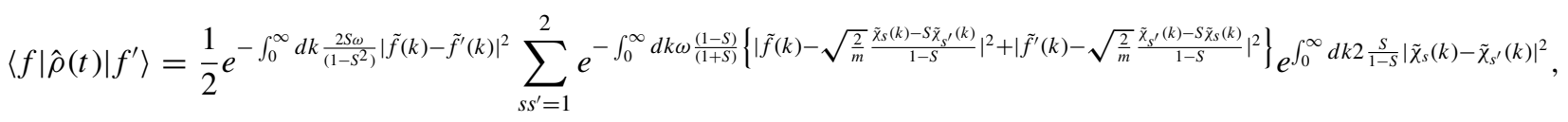

absorbing the normalization factor $\frac{2[1-S]}{\pi[1+S]}$ in the functional integration element, $D f \equiv \prod_{k>0} d \tilde{f}_{R}(k) d \tilde{f}_{I}(k) \frac{2[1-S]}{\pi[1+S]} \omega(k) d k$.

This can be written in terms of $f(x)$ as follows. First, note that $S$ depends upon $\omega$, so write it explicitly $S(\omega)$ and then make the approximation $S(\omega) \approx S(m)$, where $S$ multiplies $\tilde{\chi}_{s}(k)$, since $\tilde{\chi}_{s}(k)$ has nonrelativistic momenta. This cannot be done where $S$ multiplies $\tilde{f}(k)$. Then, upon taking the Fourier transform, note that $\omega$ becomes a differential operator, $\omega \rightarrow \hat{\omega}=\left[m^{2}-\right.$ $\left.d^{2} / d x^{2}\right]^{1 / 4}$, and similarly $S(\omega)$ becomes a differential operator, $S(\omega) \rightarrow \hat{S}=S(\hat{\omega})$. The result is

$$
\begin{aligned}
\left\langle f|\hat{\rho}(t)| f^{\prime}\right\rangle \approx & \frac{1}{2} e^{-\int_{-\infty}^{\infty} d x\left\{\left[\frac{\hat{\omega} \hat{S}}{\left(1-S^{2}\right)}\right]^{1 / 2}\left[f(x)-f^{\prime}(x)\right]\right\}^{2}} \sum_{s s^{\prime}=1}^{2} e^{-\frac{1}{2} \int_{-\infty}^{\infty} d x\left\{\left[\hat{\omega} \frac{(1-\hat{S})}{(1+S)}\right]^{1 / 2}\left[f(x)-\sqrt{\frac{2}{m}} \frac{x_{s}(x)-S(m) x_{s^{\prime}}(x)}{1-S(m)}\right]\right\}^{2}} \\
& \times e^{-\frac{1}{2} \int_{-\infty}^{\infty} d x\left\{\left[\hat{\omega} \frac{(1-\hat{S})}{(1+\hat{S})}\right]^{1 / 2}\left[f^{\prime}(x)-\sqrt{\frac{2}{m}} \frac{\chi_{S^{\prime}}(x)-S(m) x_{s}(x)}{1-S(m)}\right]\right\}^{2}} e^{\int_{-\infty}^{\infty} d x \frac{S(m)}{1-S(m)}\left[\chi_{s}(x)-\chi_{s^{\prime}}(x)\right]^{2}}
\end{aligned}
$$

Equations (5.1)-(5.3) have the same general form as Eqs. (4.1) and (4.2), except for the last exponential factor in Eqs. (5.1)-(5.3): this factor ensures that $\operatorname{Tr} \hat{\rho}(t)=\operatorname{Tr} \hat{\rho}(0)$ for the more complicated time dependence of these expressions.

The first (decaying) exponential factor in Eq. (5.3) is large only if $f(x) \approx f^{\prime}(x)$ : this characterizes the decay to eigenstates of $\hat{\phi}(x)$. For $s=s^{\prime}$, the second two exponentials are large if also $f(x) \approx f^{\prime}(x)$, and approaches the mass density distribution $\sqrt{\frac{2}{m}} \chi_{s}(x)$. Since these conditions can all be satisfied, their density matrix contribution can be large.

If $s \neq s^{\prime}$, the density matrix must decay, since both of these exponentials cannot be large since $f(x), f^{\prime}(x)$ have to approach different mass distributions when $\chi_{s}(x) \neq \chi_{s^{\prime}}(x)$. 
In fact, for short times, using $1 \gg S \approx 0$, using Eq. (A13), expressions (5.2) and (5.3) become identical to (4.2), the collapse expression when $H=0$.

For large times, $1-S(\omega) \rightarrow \frac{2 \omega}{\lambda t}$ and, using (A14), the limit of (5.2) and (5.3) is

$$
\begin{aligned}
\left\langle f|\hat{\rho}(t)| f^{\prime}\right\rangle & =\frac{1}{2} e^{-\frac{\lambda t}{2} \int_{0}^{\infty} d k\left|\tilde{f}(k)-\tilde{f}^{\prime}(k)\right|^{2}} \sum_{s s^{\prime}=1}^{2} e^{\int_{0}^{\infty} d k \sqrt{2 m} \operatorname{Re}\left[\tilde{f}(k)-\tilde{f}^{\prime}(k)\right]\left[\tilde{\chi}_{s}(k)-\tilde{\chi}_{s^{\prime}}(k)\right]^{*}} e^{-\frac{1}{\lambda t} \int_{0}^{\infty} d k \omega^{2}\left[|\tilde{f}(k)|^{2}+\left|\tilde{f}^{\prime}(k)\right|^{2}\right]} e^{-\int_{0}^{\infty} d k\left|\tilde{\chi}_{s}(k)-\tilde{\chi}_{s^{\prime}}(k)\right|^{2}} \\
& =\frac{1}{2} e^{-\frac{\lambda t}{4} \int_{-\infty}^{\infty} d x\left[f(x)-f^{\prime}(x)\right]^{2}} \sum_{s s^{\prime}=1}^{2} e^{\sqrt{\frac{m}{2}} \int_{-\infty}^{\infty} d x\left[f(x)-f^{\prime}(x)\right]\left[\chi_{s}(x)-\chi_{s^{\prime}}(x)\right]} e^{-\frac{1}{2 \lambda t} \int_{-\infty}^{\infty} d x\left[(\hat{\omega} f)^{2}(x)+\left(\hat{\omega} f^{\prime}\right)^{2}(x)\right]} e^{-\frac{1}{2} \int_{-\infty}^{\infty} d x\left[\chi_{s}(x)-\chi_{s^{\prime}}(x)\right]^{2}} .
\end{aligned}
$$

Note that the long time approximation is dependent on the mode. In order to apply it to all modes as we have done here, we are implicitly assuming that $f$ and $f^{\prime}$ are chosen to be sufficiently smooth so that $1-S(\omega) \sim \frac{2 \omega}{\lambda t}$ is valid for all component modes.

For the diagonal terms, $s=s^{\prime}$, clearly the first exponent dominates. There is no dependence upon the wave functions. The first exponential on the last line ensures the proper trace (were it not there, the trace would be infinite).

It is shown in the Appendix, Eq. (A15), that this asymptotic behavior can be explained as each mode acting like a thermal density matrix $C e^{-\frac{1}{k_{B} T} \hat{H}}$, where $\hat{H}=\omega\left[a_{k}^{\dagger} a_{k}+b_{k}^{\dagger} b_{k}\right]$, and with the temperature increasing with time, $\frac{\lambda t}{2 \omega}=\frac{1}{e^{\frac{\omega}{k_{B} T}}-1}$. This is the eventual domination of the particle creation, caused by collapse to field eigenstates.

For the off-diagonal terms, $s \neq s^{\prime}$, really the same holds true. There is dependence on the wave function in the second exponential but, because the first exponential is only large if $f(x) \approx f^{\prime}(x)$, this forces the second exponential toward the value 1 , negating the apparent dependence upon the wave function. (The wave function dependence in the last line has no time dependence; it is just the initial trace.)

\section{CONCLUDING REMARKS}

In standard quantum theory, the state vector does not describe what actually happens, the occurrence of events. If that is to be modified by CSL so that description does occur, degrees of freedom have an increase of energy due to the CSL-induced narrowing of wave functions.

In the classical physics lexicon, the world is made out of two kinds of things, particles and fields. In quantum theory, the two ideas merge, since each can be written in terms of the other. Particles have a finite number of degrees of freedom, while fields have an infinite number of degrees of freedom.

Thus, the CSL-induced energy increase associated with particles is finite (and, in nonrelativistic CSL small enough that it has not yet experimentally either been found or found not to exist), while the energy increase associated with fields is infinite, and therefore experimentally ruled out. If the CSL collapse mechanism is chosen by nature, it is a choice of particles over fields. The point of this paper has been to give the details of the road not taken.

\section{APPENDIX: HARMONIC OSCILLATOR AND COLLAPSE GENERATED BY $\hat{X}$}

Here we give the solution of part of the Lindblad equation (1.6), the harmonic oscillator when the collapse-generating operator is in position:

$$
\frac{d}{d t} \hat{\rho}(t)=-i \omega\left[\hat{X}^{2}+\frac{1}{4} \hat{P}^{2}, \hat{\rho}(t)\right]-\frac{\lambda}{\omega}[\hat{X},[\hat{X}, \hat{\rho}(t)]]
$$

with the initial condition

$$
\hat{\rho}(0)=e^{-\frac{1}{2} \gamma_{1}^{2}} e^{\gamma_{1} \hat{a}^{\dagger}}|0\rangle\langle 0| e^{\gamma_{2} \hat{a}} e^{-\frac{1}{2} \gamma_{2}^{2}},
$$

and $\gamma_{1}, \gamma_{2}$ real.

Note, $\hat{\rho}^{\dagger}(0) \neq \hat{\rho}(0)$, and $\operatorname{Tr} \hat{\rho}(0)=e^{-\frac{1}{2}\left[\gamma_{1}-\gamma_{2}\right]^{2}} \neq 1$ (unless $\left.\gamma_{1}=\gamma_{2}\right): \hat{\rho}(0)$ is part of the initial density matrix utilized in Sec. V, which of course is Hermitian and trace 1. Comparing the Hamiltonian to that of the usual harmonic oscillator, $\frac{1}{2} M \Omega^{2} \hat{X}^{2}+\frac{1}{2 M} \hat{P}^{2}$, the relation is $M=\frac{2}{\omega}, \Omega=\omega$, and so the annihilation operator is $\hat{a}=\sqrt{\frac{M \Omega}{2}} \hat{X}+i \sqrt{\frac{1}{2 M \Omega}} \hat{P}=\hat{X}+i \frac{1}{2} \hat{P}$.

Equation (A1), expressed in terms of creation and annihilation operators, is

$$
\frac{d}{d t} \hat{\rho}(t)=-i \omega\left[\hat{a}^{\dagger} \hat{a}, \hat{\rho}(t)\right]-\frac{\lambda}{4 \omega}\left[\hat{a}^{\dagger}+\hat{a},\left[\hat{a}^{\dagger}+\hat{a}, \hat{\rho}(t)\right] .\right.
$$

We first will solve (A3) for $\hat{\rho}(t)$ subject to the initial condition (A2), and then proceed to find $\left\langle X|\hat{\rho}(t)| X^{\prime}\right\rangle$, which is utilized in Sec. V.

From (A3), we find the equations for the expectation values of $\hat{a}, \hat{a}^{\dagger}, \hat{a}^{\dagger} \hat{a}, \hat{a}^{2}, \hat{a}^{\dagger 2}$, where, e.g., $\overline{\hat{a}^{\dagger} \hat{a}(t)} \equiv \operatorname{Tr} \hat{a}^{\dagger} \hat{a} \rho(t)$ :

$$
\begin{aligned}
\frac{d}{d t} \overline{\hat{a}(t)} & =-i \omega \operatorname{Tr} \rho(t)\left[\hat{a}, \hat{a}^{\dagger} \hat{a}\right]=-i \omega \overline{\hat{a}(t)}, \text { so } \\
\overline{\hat{a}(t)} & =\gamma_{1} \operatorname{Tr} \hat{\rho}(0) e^{-i \omega t} \\
\overline{\hat{a}^{\dagger}(t)} & =\gamma_{2} \operatorname{Tr} \hat{\rho}(0) e^{i \omega t} \\
\frac{d}{d t} \overline{\hat{a}^{\dagger} \hat{a}(t)} & =-\frac{\lambda}{4 \omega} \operatorname{Tr} \hat{\rho}(t)\left[\hat{a}^{\dagger}+\hat{a},\left[\hat{a}^{\dagger}+\hat{a}, \hat{a}^{\dagger} \hat{a}\right]\right. \\
& =\frac{\lambda}{2 \omega} \operatorname{Tr} \hat{\rho}(0) \text { so } \overline{\hat{a}^{\dagger} \hat{a}(t)}=\left[\frac{\lambda}{2 \omega} t+\gamma_{1} \gamma_{2}\right] \operatorname{Tr} \hat{\rho}(0),
\end{aligned}
$$




$$
\begin{aligned}
\frac{d}{d t} \overline{\hat{a}^{2}(t)} & =-\frac{\lambda}{2 \omega} \operatorname{Tr} \hat{\rho}(0)-2 i \omega \overline{\hat{a}^{2}(t)}, \text { so } \\
\overline{\hat{a}^{2}(t)} & =\left[-\frac{\lambda}{2 \omega} e^{-i \omega t} \frac{\sin \omega t}{\omega}+\gamma_{1}^{2} e^{-2 i \omega t}\right] \operatorname{Tr} \hat{\rho}(0), \\
\overline{\hat{a}^{\dagger 2}(t)} & =\left[-\frac{\lambda}{2 \omega} e^{i \omega t} \frac{\sin \omega t}{\omega}+\gamma_{2}^{2} e^{2 i \omega t}\right] \operatorname{Tr} \hat{\rho}(0) .
\end{aligned}
$$

These expectation values determine $\hat{\rho}(t)$, when we make the ansatz of the following quadratic form:

$$
\hat{\rho}(t)=C(t) e^{R(t) \hat{a}^{\dagger 2}} e^{\beta_{1}(t) \hat{a}^{\dagger}} e^{S(t) \hat{a}_{L}^{\dagger} \hat{a}_{R}}|0\rangle\langle 0| e^{\beta_{2}^{*}(t) \hat{a}} e^{R^{*}(t) \hat{a}^{2}},
$$

where

$$
e^{S(t) \hat{a}_{L}^{\dagger} \hat{a}_{R}}|0\rangle\left\langle 0\left|=\sum_{n=0}^{\infty} \frac{S^{n}(t)}{n !} \hat{a}^{\dagger n}\right| 0\right\rangle\langle 0| \hat{a}^{n} .
$$

It follows from commuting the exponentials past $\hat{a}, \hat{a}^{\dagger}$ that

$$
\begin{aligned}
\hat{a} \hat{\rho}(t) & =2 R(t) \hat{a}^{\dagger} \hat{\rho}(t)+S(t) \hat{\rho}(t) \hat{a}+\beta_{1}(t) \hat{\rho}(t), \\
\hat{\rho} \hat{a}^{\dagger} & =2 R^{*}(t) \hat{\rho}(t) \hat{a}+S(t) \hat{a}^{\dagger} \hat{\rho}(t)+\beta_{2}^{*}(t) \hat{\rho}(t),
\end{aligned}
$$

using

$$
\begin{aligned}
\hat{a} e^{S(t) \hat{a}_{L}^{\dagger} \hat{a}_{R}}|0\rangle\langle 0| & =\sum_{n=0}^{\infty} \frac{S^{n}(t)}{n !} \hat{a} \hat{a}^{\dagger n}|0\rangle\langle 0| \hat{a}^{n} \\
& =S(t) \sum_{n=1}^{\infty} \frac{S^{n-1}(t)}{(n-1) !} \hat{a}^{\dagger(n-1)}|0\rangle\langle 0| \hat{a}^{n-1} \hat{a} \\
& =S(t) e^{S(t) \hat{a}_{L}^{\dagger} \hat{a}_{R}}|0\rangle\langle 0| \hat{a} .
\end{aligned}
$$

Then it follows from taking the trace of (A6) and products of operators with (A6) that

$$
\begin{aligned}
\overline{\hat{a}(t)} & =2 R(t) \overline{\hat{a}^{\dagger}(t)}+S(t) \overline{\hat{a}(t)}+\beta_{1}(t) \operatorname{Tr} \hat{\rho}(0), \\
\overline{\hat{a}^{\dagger}(t)} & =2 R^{*}(t) \overline{\hat{a}(t)}+S(t) \overline{\hat{a}^{\dagger}(t)}+\beta_{2}^{*}(t) \operatorname{Tr} \hat{\rho}(0), \\
\overline{\hat{a}^{\dagger} \hat{a}(t)} & =2 R(t) \overline{\hat{a}^{\dagger 2}(t)}+S(t)\left[\operatorname{Tr} \hat{\rho}(0)+\overline{\hat{a}^{\dagger} \hat{a}(t)}\right]+\beta_{1}(t) \overline{\hat{a}^{\dagger}(t)} \\
& =2 R^{*}(t) \overline{\hat{a}^{2}(t)}+S(t)\left[\operatorname{Tr} \hat{\rho}(0)+\overline{\hat{a}^{\dagger} a(t)}\right]+\beta_{2}^{*}(t) \overline{\hat{a}(t)},
\end{aligned}
$$

(A7c)

$$
\overline{\hat{a}^{2}(t)}=2 R(t)\left[\operatorname{Tr} \hat{\rho}(0)+\overline{\hat{a}^{\dagger} \hat{a}(t)}\right]+S(t) \overline{\hat{a}^{2}(t)}+\beta_{1}(t) \overline{\hat{a}(t)},
$$

$\overline{\hat{a}^{\dagger 2}(t)}=2 R^{*}(t)\left[\operatorname{Tr} \hat{\rho}(0)+\overline{\hat{a}^{\dagger} \hat{a}(t)}\right]+S(t) \overline{\hat{a}^{\dagger 2}(t)}+\beta_{2}^{*}(t) \overline{\hat{a}^{\dagger}(t)}$.

The solution of Eqs. (A7a)-(A7e), using (A4a)-(A4e), is

$$
\begin{aligned}
S(t) & =1-\frac{\frac{\lambda}{2 \omega} t+1}{\left[\frac{\lambda}{2 \omega} t+1\right]^{2}-\left[\frac{\lambda}{2 \omega^{2}} \sin \omega t\right]^{2}}, \\
R(t) & =\frac{-\frac{\lambda}{4 \omega^{2}} e^{-i \omega t} \sin \omega t}{\left[\frac{\lambda}{2 \omega} t+1\right]^{2}-\left[\frac{\lambda}{2 \omega^{2}} \sin \omega t\right]^{2}}, \\
\beta_{1}(t) & =\frac{\left[\frac{\lambda}{2 \omega} t+1\right] \gamma_{1} e^{-i \omega t}+\lambda \gamma_{2} \frac{1}{2 \omega^{2}} \sin \omega t}{\left[\frac{\lambda}{2 \omega} t+1\right]^{2}-\left[\frac{\lambda}{2 \omega^{2}} \sin \omega t\right]^{2}}, \\
\beta_{2}^{*}(t) & =\frac{\left[\frac{\lambda}{2 \omega} t+1\right] \gamma_{2} e^{i \omega t}+\lambda \gamma_{1} \frac{1}{2 \omega^{2}} \sin \omega t}{\left[\frac{\lambda}{2 \omega} t+1\right]^{2}-\left[\frac{\lambda}{2 \omega^{2}} \sin \omega t\right]^{2}} .
\end{aligned}
$$

We now make some approximations. The characteristic time for the oscillations is $2 \pi / \omega<2 \pi / m \equiv \tau \approx 3 \times$ $10^{-23} \mathrm{~s}$ for a nucleon (a timescale very short compared to the collapse time). Therefore, after say $t \approx 100 \tau$, since $\frac{\lambda}{2 \omega^{2}} \sin \omega t<\frac{\lambda}{2 \omega^{2}}<<\frac{\lambda}{2 \omega} t$, we may neglect the $\sin \omega t$ term, obtaining

$$
\begin{aligned}
S(t) & \approx \frac{\frac{\lambda}{2 \omega} t}{\frac{\lambda}{2 \omega} t+1}, \\
R(t) & \approx 0, \\
\beta_{1}(t) & =\frac{\gamma_{1} e^{-i \omega t}}{\frac{\lambda}{2 \omega} t+1}, \\
\beta_{2}^{*}(t) & =\frac{\gamma_{2} e^{i \omega t}}{\frac{\lambda}{2 \omega} t+1} .
\end{aligned}
$$

Moreover, the exponentials $e^{ \pm i \omega t}$ multiply $\gamma_{i}$. In our problem, $\gamma_{i} \sim \tilde{\chi}(k)$, which essentially vanishes unless $k \ll m$. So, we may set $e^{ \pm i \omega t} \approx e^{ \pm i m t}$. In that case, we may consider the solution only at times which are integer multiples of $\tau$, since these are so closely spaced on the collapse dynamics timescale. Therefore, so far we have

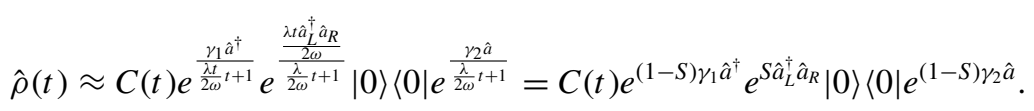

It remains to find $C(t)$. Since, according to (A3), $\frac{d}{d t} \operatorname{Tr} \hat{\rho}(t)=0$, it follows from (A10) that

$$
\begin{aligned}
& 0=\frac{1}{C(t)} \dot{C}(t) \operatorname{Tr} \hat{\rho}(0)+\overline{\hat{a}^{\dagger}(t)} \frac{d}{d t} \frac{\gamma_{1}}{\frac{\lambda}{2 \omega} t+1}+\left[\operatorname{Tr} \hat{\rho}(0)+\overline{\hat{a}^{\dagger} \hat{a}(t)}\right] \frac{d}{d t} \frac{\frac{\lambda t}{2 \omega}}{\frac{\lambda}{2 \omega} t+1}+\overline{\hat{a}(t)} \frac{d}{d t} \frac{\gamma_{2}}{\frac{\lambda}{2 \omega} t+1} \text { or } \\
& 0=\frac{1}{C(t)} \dot{C}(t)+\frac{\frac{\lambda}{2 \omega}}{\frac{\lambda}{2 \omega} t+1}-\gamma_{1} \gamma_{2} \frac{\frac{\lambda}{2 \omega}}{\left[\frac{\lambda}{2 \omega} t+1\right]^{2}} \quad \text { with solution } \\
& C(t)=\frac{1}{\frac{\lambda}{2 \omega} t+1} e^{\frac{\gamma_{1} \gamma_{2} \frac{\lambda}{2 \omega} t}{2 \omega} t+1} e^{-\frac{1}{2}\left[\gamma_{1}^{2}+\gamma_{2}^{2}\right]}=(1-S) e^{S \gamma_{1} \gamma_{2}} e^{-\frac{1}{2}\left[\gamma_{1}^{2}+\gamma_{2}^{2}\right]} \text {. }
\end{aligned}
$$




\section{Density matrix in the position representation}

We now proceed to calculate the matrix element of (A10), using $e^{S \hat{a}_{L}^{\dagger} \hat{a}_{R}}|0\rangle\left\langle 0\left|=\sum_{n=0}^{\infty} S^{n}\right| n\right\rangle\langle n|$, whose matrix elements in the position representation are given by a well-known identity involving Hermite polynomials [9]. We also use the Campbell-Baker-Haussdorf theorem, obtaining

$$
\begin{aligned}
\left\langle X|\hat{\rho}(t)| X^{\prime}\right\rangle= & C(t) e^{(1-S) \gamma_{1}\left[X-\frac{\partial}{2 \partial X}\right]} e^{(1-S) \gamma_{2}\left[X^{\prime}-\frac{\partial}{2 \partial X^{\prime}}\right]} \mid \sum_{n=0}^{\infty} S^{n}\langle X \mid n\rangle\left\langle X^{\prime} \mid n\right\rangle \\
= & C(t) e^{-\frac{1}{4}(1-S)^{2} \gamma_{1}^{2}} e^{-\frac{1}{4}(1-S)^{2} \gamma_{2}^{2}} e^{(1-S) \gamma_{1} X} e^{(1-S) \gamma_{2} X^{\prime}} e^{-(1-S) \gamma_{1} \frac{\partial}{2 \partial X}} e^{-(1-S) \gamma_{2} \frac{\partial}{2 \partial X^{\prime}}} \frac{\sqrt{2}}{\sqrt{\pi\left[1-S^{2}\right]}} e^{-\frac{1-S}{2(1+S)}\left[X+X^{\prime}\right]^{2}} e^{-\frac{1+S}{2(1-S)}\left[X-X^{\prime}\right]^{2}} \\
= & \sqrt{\frac{2[1-S]}{\pi[1+S]}} e^{S \gamma_{1} \gamma_{2}} e^{-\frac{1}{2}\left[\gamma_{1}^{2}+\gamma_{2}^{2}\right]} e^{-\frac{1}{4}(1-S)^{2} \gamma_{1}^{2}} e^{-\frac{1}{4}(1-S)^{2} \gamma_{2}^{2}} e^{(1-S) \gamma_{1} X} e^{(1-S) \gamma_{2} X^{\prime}} \\
& \times e^{-\frac{1-S}{2(1+S)}\left[X+X^{\prime}-\frac{1-S}{2}\left(\gamma_{1}+\gamma_{2}\right)\right]^{2}} e^{-\frac{1+S}{2(1-S)}\left[X-X^{\prime}-\frac{1-S}{2}\left(\gamma_{1}-\gamma_{2}\right)\right]^{2}} \\
= & \sqrt{\frac{2[1-S]}{\pi[1+S]}} e^{-\frac{2 S}{\left(1-S^{2}\right)}\left[X-X^{\prime}\right]^{2}} e^{-\frac{1-S}{(1+S)}\left\{\left[X-\frac{\gamma_{1}-S \gamma_{2}}{1-S}\right]^{2}+\left[X^{\prime}-\frac{\gamma_{2}-S \gamma_{1}}{1-S}\right]^{2}\right\}} e^{\frac{S}{1-S}\left[\gamma_{1}-\gamma_{2}\right]^{2}}
\end{aligned}
$$

\section{Short time and long time limits}

For short times, $S \approx \frac{\lambda}{2 \omega} t \ll 1$, (A12) becomes

$$
\left\langle X|\hat{\rho}(t)| X^{\prime}\right\rangle \approx \sqrt{\frac{2}{\pi}} e^{-\frac{\lambda t}{\omega}\left[X-X^{\prime}\right]^{2}} e^{-\left[X-\gamma_{1}\right]^{2}} e^{-\left[X^{\prime}-\gamma_{2}\right]^{2}} .
$$

It is consistent to neglect the exponent in the last factor in Eq. (A12), $e^{\frac{S}{1-S}\left[\gamma_{1}-\gamma_{2}\right]^{2}} \approx e^{-\frac{\lambda t}{2 \omega}\left[\gamma_{1}-\gamma_{2}\right]^{2}} \approx 1$ to accompany the approximation $S \gamma_{i}<<\gamma_{i}$, so that, even in this approximation, the property that the trace is unchanged is preserved, $\operatorname{Tr} \hat{\rho}(t)=$ $\operatorname{Tr} \hat{\rho}(0)=e^{-\frac{1}{2}\left[\gamma_{1}-\gamma_{2}\right]^{2}}$.

For long times, using $1-S \approx \frac{2 \omega}{\lambda t}$, (A12) becomes

$$
\left\langle X|\hat{\rho}(t)| X^{\prime}\right\rangle \approx \sqrt{\frac{2 \omega}{\pi \lambda t}} e^{-\frac{\lambda t}{2 \omega}\left[X-X^{\prime}\right]^{2}} e^{\left[X-X^{\prime}\right]\left[\gamma_{1}-\gamma_{2}\right]} e^{-\frac{\omega}{\lambda t}\left[X^{2}+X^{\prime 2}\right]} e^{-\frac{1}{2}\left[\gamma_{1}-\gamma_{2}\right]^{2}} .
$$

The first exponential dominates: it is large only if $\left|X-X^{\prime}\right|^{2} \sim \frac{2 \omega}{\lambda t}$, in which case the second and third exponentials approach 1 as $t \rightarrow \infty$. The third and fourth exponentials are there to preserve the property that the trace is unchanged.

To understand this behavior better, return to Eq. (A10), where the $(1-S) \gamma_{i} \rightarrow \gamma_{i} \frac{2 \omega}{\lambda t}$ dependence makes it clear that the $\gamma_{i}$ dependence is asymptotically negligible. Then, concentrate on the Trace 1 remainder:

$$
\begin{aligned}
\hat{\rho}(t) & =(1-S) e^{S \hat{a}_{L}^{\dagger} \hat{a}_{R}}|0\rangle\left\langle 0\left|=(1-S) \sum_{n=1}^{\infty} S^{n}\right| n\right\rangle\langle n| \\
& =(1-S) \sum_{n=1}^{\infty} e^{n \ln S}|n\rangle\left\langle n\left|=(1-S) e^{\hat{a}^{\dagger} \hat{a} \ln S} \sum_{n=1}^{\infty}\right| n\right\rangle\langle n| \\
& =(1-S) e^{\hat{a}^{\dagger} \hat{a} \ln S} .
\end{aligned}
$$

This is a thermal density matrix, with identification of $S=e^{-\frac{\omega}{k_{B} T}}$, with temperature $T$ (and $k_{B}$ the Boltzmann constant) and Hamiltonian $=\omega \hat{a}^{\dagger} \hat{a}$. The mean particle number is $\operatorname{Tr} \hat{a}^{\dagger} \hat{a} \rho(t)=\frac{S}{1-S}=\frac{\lambda t}{2 \omega}=\frac{1}{e^{\frac{\omega}{e_{B}}}-1}$. So, the particle number increase with increasing time is the same as the occupation number increase with increasing temperature of a harmonic oscillator in a thermal bath, always in thermal equilibrium.

\section{Harmonic oscillator and collapse generated by $\hat{p}$}

The other harmonic oscillator problem in Eq. (1.6),

$$
\frac{d}{d t} \hat{\rho}(t)=-i \omega\left[\frac{1}{4} \hat{x}^{2}+\hat{p}^{2}, \hat{\rho}(t)\right]-\frac{\lambda}{\omega}[\hat{p},[\hat{p}, \hat{\rho}(t)]]=-i \omega\left[\hat{a}^{\dagger} \hat{a}, \rho(t)\right]-\frac{\lambda}{4 \omega}\left[\hat{a}^{\dagger}+\hat{a},\left[\hat{a}^{\dagger}+\hat{a}, \hat{\rho}(t)\right]\right]
$$

subject to the initial condition

$$
\hat{\rho}(0)=e^{-\frac{1}{2} \gamma_{1}^{\prime 2}} e^{\gamma_{1}^{\prime} \hat{a}^{\dagger}}|0\rangle\langle 0| e^{\gamma_{2}^{\prime} \hat{a}} e^{-\frac{1}{2} \gamma_{2}^{\prime 2}},
$$


has precisely the same solution:

$$
\left\langle p|\rho(t)| p^{\prime}\right\rangle=\sqrt{\frac{2[1-S]}{\pi[1+S]}} e^{-\frac{2 S}{\left(1-S^{2}\right)}\left[p-p^{\prime}\right]^{2}} e^{-\frac{1-S}{(1+S)}\left\{\left[p-\frac{\gamma_{1}^{\prime}-S \gamma_{2}^{\prime}}{1-S}\right]^{2}+\left[p^{\prime}-\frac{\gamma_{2}^{\prime}-S \gamma_{1}^{\prime}}{1-S}\right]^{2}\right\}} e^{\frac{S}{1-S}\left[\gamma_{1}^{\prime}-\gamma_{2}^{\prime}\right]^{2}} .
$$

[1] P. Pearle, Combining stochastic dynamical state-vector reduction with spontaneous localization, Phys. Rev. A 39, 2277 (1989).

[2] G. C. Ghirardi, P. Pearle, and A. Rimini, Markov processes in Hilbert space and continuous spontaneous localization of systems of identical particles, Phys. Rev. A 42, 78 (1990).

[3] E. Schrödinger, Die gegenwartige Situation in der Quantenmechanik (The Present Status of Quantum Mechanics), Die Naturwissenschaften 23, pp. 807-812, 823-828, 844-849 (1935).

[4] The Stanford Encyclopedia of Philosophy, https://plato.stanford. edu, contains discussions and references in their articles entitled Bohmian Mechanics, Many-Worlds interpretation of Quantum Mechanics, The Consistent Histories Approach to Quantum Mechanics, and The Role of Decoherence in Quantum Mechanics.

[5] G. C. Ghirardi and A. Bassi, Dynamical reduction models, Phys. Rep. 379, 257 (2003).

[6] A. Bassi, K. Lochan, S. Satin, T. Singh, and H. Ulbricht, Models of wave function collapse, underlying theories, and experimental tests, Rev. Mod. Phys. 85, 471 (2013).
[7] P. Pearle, Toward a relativistic theory of statevector reduction, in Sixty-Two Years of Uncertainty, edited by A. Miller (Plenum, New York, 1990), p. 193.

[8] G. C. Ghirardi, R. Grassi, and P. Pearle, Relativistic dynamical reduction models: General framework and examples, Found. Phys. 20, 1271 (1990).

[9] The identity (e.g., in the last section of Wikipedia's article on Hermite polynomials) is

$$
\sum_{n=0}^{\infty} S^{n} \psi_{n}(x) \psi_{n}(y)=\frac{1}{\sqrt{\pi\left[1-S^{2}\right]}}\left[e^{-\frac{1-S}{1+S} \frac{(x+y)^{2}}{4}}+e^{-\frac{1+S}{1-S} \frac{(x-y)^{2}}{4}}\right],
$$

where $\psi_{n}(x)$ is the usual harmonic oscillator wave function. However, the usual annihilation operator is $a=\frac{1}{\sqrt{2}}[\hat{X}+i \hat{P}]$ whereas the one used here is $a=\left[\hat{X}+i \frac{1}{2} \hat{P}\right]$. This means that the wave functions $\langle X \mid n\rangle=2^{1 / 4} \psi_{n}(\sqrt{2} X)$. 